\title{
Anisotropic silica mesostructures for DNA encapsulation
}

\author{
APARNA GANGULY and ASHOK K GANGULI* \\ Department of Chemistry, Indian Institute of Technology, HauzKhas, New Delhi 110 016, India
}

MS received 28 March 2012

\begin{abstract}
The encapsulation of biomolecules in inert meso or nanostructures is an important step towards controlling drug delivery agents. Mesoporous silica nanoparticles (MSN) are of immense importance owing to their high surface area, large pore size, uniform particle size and chemical inertness. Reverse micellar method with CTAB as the surfactant has been used to synthesize anisotropic mesoporous silica materials. We have used the anisotropic silica nanostructures for DNA encapsulation studies and observed a loading capacity of $\sim 8 \mu \mathrm{g} \mathrm{mg}^{-1}$ of the sample. On functionalizing the pores of silica with amine group, the amount of DNA loaded on the rods decreases which is due to a reduction in the pore size upon grafting of amine groups.
\end{abstract}

Keywords. Surfactant; reverse micellar method; silica mesostructures; surface area.

\section{Introduction}

Amorphous silica nanoparticles are important (Choy et al 2000) owing to their high chemical resistance to microbial attack, low cost and toxicity, thermal stability and ease of surface modification. Silica nanoparticles also have the advantage of incorporating other inorganic materials for magnetic or optical properties. Application of mesoporous silica materials conjugated to DNA lies in its use as a non-viral vector for gene delivery (Kumar et al 2004; Radu et al 2004; Roy et al 2005). The prospect of gene therapy and genetic engineering adds a new vision to the existing medical techniques for transferring custom genetic material into cellular environments to rectify genetic diseases. At present only a few methods are practiced to deliver and protect DNA during cellular transfection. Of these inorganic nanoparticles, mesoporous silica forms the most popular choice. These materials are characterized by large surface areas (in excess of $1000 \mathrm{~m}^{2} / \mathrm{g}$ ) and large internal pore volumes and narrow pore size distributions that can be tailored during synthesis. These characteristics have led to the use of mesoporous silica material as a potential delivery agent for guest molecules, which can be easily adsorbed into their pores by ion exchange or covalent bonding for later release within cells (Kisler et al 2001; Deere et al 2001).

Increased uptake capacities (as compared to non-porous materials) and subsequent release of the adsorbed materials has been observed for these mesoporous materials. It is well known that a silica surface cannot adsorb duplex DNA in common aqueous solution because of the electrostatic repulsion of the silica surface and polyanionic DNA (Fujiwara et al 2005). However duplex DNA in phosphoric acid form can be easily adsorbed in an aqueous solution.

\footnotetext{
*Author for correspondence (ashok@ chemistry.iitd.ac.in)
}

MSNs (mesoporous silica nanoparticles) have been used to load plasmid RNA (1-1000 kbp, a straight length of $340 \mathrm{~nm}$ ) and siRNA (small interfering RNA; 21-25 bp, a straight length of $7-8.5 \mathrm{~nm}$ ), but both plasmid RNA and siRNA were loaded only on the external surface of the pre-modified (polycation such as PEI) MSNs (Zhu et al 2005; Torney et al 2007; Chen et al 2009; Meng et al 2010).

Solberg and Landry (2006) have shown that amine functionalized mesoporous materials exhibit advanced adsorption properties for DNA immobilization, due to their large surface areas and internal pore volume and a narrow pore size distribution. They have also reported that the adsorption of DNA is dependent on the type of metal cation used to mediate the charge between the DNA molecule and the silica surface. A higher affinity of the DNA is observed for $\mathrm{Mg}^{2+}$ than either $\mathrm{Ca}^{2+}$ or $\mathrm{Na}^{+}$(Solberg and Landry 2006). The amine functionalized silica nanoparticles provides stability to the plasmid DNA against cleavage. Also the released plasmids were found to be biologically active (He et al 2003). Gao et al 2009 have used fluorocarbon (FC) surfactant to create silica nanospheres with large pores and studied the encapsulation of firefly luciferase plasmid DNA in these mesostructures (Gao et al 2009). On functionalizing the pores, the amount of DNA adsorbed was found to increase drastically. Silica-based nano-sized materials with cationic groups have been reported to electrostatically bind DNA molecules and to undergo gene delivery successfully (He et al 2003; Pearce et al 2008).

However, all the above reports have used spherical mesoporous silica materials for the encapsulation of DNA and studying its efficiency as a drug delivery vector. In this paper, we report the use of anisotropic silica nanostructures for the encapsulation of DNA with the aim that a larger quantity of DNA can be loaded per milligram of the silica sample. Also these anisotropic nanostructures can act as a better 
delivery vehicle targeted at the diseased site with better efficiency.

\section{Materials and methods}

Commercially available cetyl trimethyl ammonium bromide (CTAB, Spectrochem, 99\%), 1-butanol (Qualigens, 99.5\%), iso-octane (Spectrochem, 99\%), chloroform (SRL, 99.5\%), ethanol (Merck, 99.9\%) and methanol (Qualigens, 99\%) were used in the synthesis. TEOS (tetra ethyl orthosilicate) and APTMS (amino propyl trimethoxysilane) was procured from Sigma Aldrich.

\subsection{Synthesis and characterization of nanorods}

Nanorods of mesoporous silica were synthesized using the reverse micellar method composed of cationic surfactant. Microemulsions with CTAB as surfactant, 1-butanol as cosurfactant, iso-octane as hydrocarbon phase and an aqueous phase were prepared. The constituents of the microemulsion were taken in the following weight fractions: $16.76 \%$ of CTAB, $13.90 \%$ of $n$-butanol, $59.29 \%$ of iso-octane and $10.05 \%$ of an aqueous phase. An aqueous phase was modified with the use of ethanol. TEOS and ammonia along with water forms the constituent of an aqueous phase. The white gel was separated from the surfactant system by centrifuging at high speed and washed with choloroform-methanol mixture to remove the surfactant. The sample was dried at $40{ }^{\circ} \mathrm{C}$ for $6 \mathrm{~h}$. The amount of water in the above composition can be varied to modify the dimension of the nanorod. Also changing the co-surfactant may lead to the formation of nanorods with different dimensions.

Powder X-ray diffraction studies were carried out on a Bruker D-8 advance X-ray diffractometer using Ni-filtered $\mathrm{CuK} \alpha$ radiation. IR spectra of the silica nanoparticles were recorded in the transmission mode in the range of 200$4000 \mathrm{~cm}^{-1}$ on a Nicolet Protégé 460 FTIR spectrometer using $\mathrm{KBr}$ discs. TEM was recorded on a Technai $\mathrm{G}^{2} 20$ (FEI) electron microscope operated at $200 \mathrm{kV}$. TEM specimens were prepared by loading a drop of the ultrasonically dispersed sample in ethanol on a carbon-coated copper grid and dried in air. Zeta potential was measured using a Malvern Zeta Sizer ZS 90 fitted with a $633 \mathrm{~nm}$ laser. $\mathrm{N}_{2}$ adsorption and desorption studies to determine the surface area were carried out on a Quantachrome Autosorb-1C operated at $77 \mathrm{~K}$. The as-prepared $\mathrm{SiO}_{2}$ samples were degassed at $150{ }^{\circ} \mathrm{C}$ for $4 \mathrm{~h}$ prior to the surface area measurements whereas the pore size distribution was calculated according to the Barret-Joyner-Halenda (BJH) method.

\subsection{Preparation of DNA conjugates}

The plasmid DNA, p Blue script $\mathrm{KS}^{+}$has been used in these studies. This is a double stranded circular DNA with size $\sim 2.9 \mathrm{kbp}$ and an ampicillin resistant. Ten $\mathrm{mg}$ of the dried silica material was dispersed in $1 \mathrm{ml}$ of an aqueous solution of $\mathrm{p}$ Blue script with varying concentrations ranging from 5 to $100 \mu \mathrm{g} \mathrm{ml}^{-1}$. The mesoporous material was allowed to equilibrate for $24 \mathrm{~h}$ under a constant stirring at $700 \mathrm{rpm}$ at $22{ }^{\circ} \mathrm{C}$. The sample was then centrifuged at an ultra-low temperatures $\left(\sim 10^{\circ} \mathrm{C}\right)$ and high rpm $(10,000)$ for $10 \mathrm{~min}$ to remove the suspended particles which minimizes the interference in subsequent spectrophotometric experiments caused by scattering of light. Supernatant liquid was collected and the amount of DNA left in the solution was determined spectrophotometrically at $260 \mathrm{~nm}$. The loading capacity of the material was determined by subtracting the pDNA content in the supernatant from that of the starting solution. All the experiments were carried out under sterilized conditions.

\subsection{Functionalization of the nanorods with amine groups}

$50 \mathrm{mg}$ of the as-synthesized silica mesostructure was boiled in $50 \mathrm{ml}$ of water to reactivate the surface hydroxyl groups. The sample was then collected by centrifugation and redispersed in ethanol. $50 \mu \mathrm{l}$ of amino propyl trimethoxysilane (APTMS) was added to it and refluxed for $6 \mathrm{~h}$. The sample was collected by centrifugation and washed with ethanol. The DNA conjugates were prepared by the method discussed above and the loading capacity was determined using spectrophotometric method.

\section{Results and discussion}

\subsection{DNA encapsulation in anisotropic structures}

Anisotropic silica mesostructures with high surface area and reasonably large pore volume was synthesized using reverse micellar method. The powder X-ray diffraction studies of the silica samples show the amorphous character

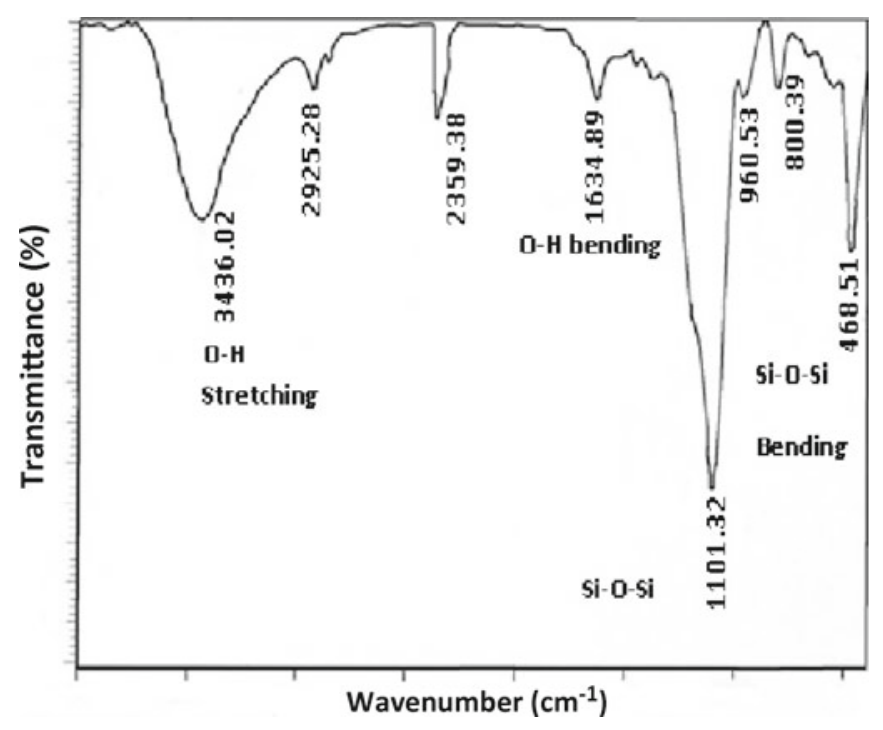

Figure 1. IR spectra of silica nanorods synthesized using CTAB as surfactant. 


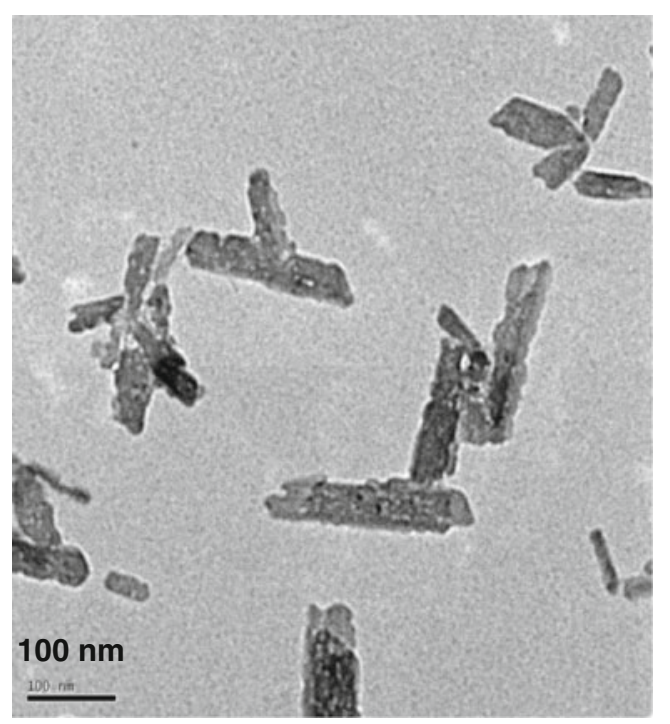

Figure 2. TEM image of silica nanorods synthesized using reverse micellar method.

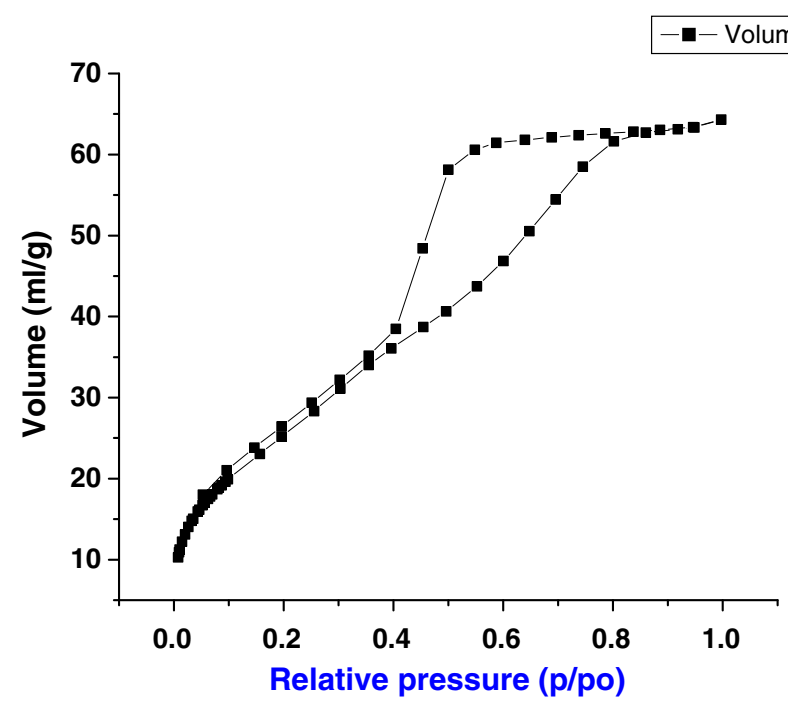

Figure 3. Nitrogen adsorption-desorption isotherm for silica nanorods.

of the sample (not shown here). The formation of silica particles was confirmed using IR spectroscopy (figure 1). TEM confirmed the formation of uniform silica nanorods with diameter $\sim 50 \mathrm{~nm}$ and length $\sim 150 \mathrm{~nm}$ (figure 2). The BET surface area measured on the degassed samples was found to be $\sim 550 \mathrm{~m}^{2} / \mathrm{g}$ and the pore volume $\sim 0.32 \mathrm{cc} / \mathrm{g}$. The $\mathrm{N}_{2}$ adsorption-desorption isotherm (figure 3) exhibits H2-type hysteresis which indicates the formation of cage-like pore structure connected with windows of smaller size (Gao et al 2009). The high pore volume favours the encapsulation of material and thus studied for DNA encapsulation. We have used double stranded $\mathrm{p}$ Blue script $\mathrm{KS}^{+}$plasmid circular DNA with size $\sim 2.9 \mathrm{kbp}$ for the encapsulation studies.

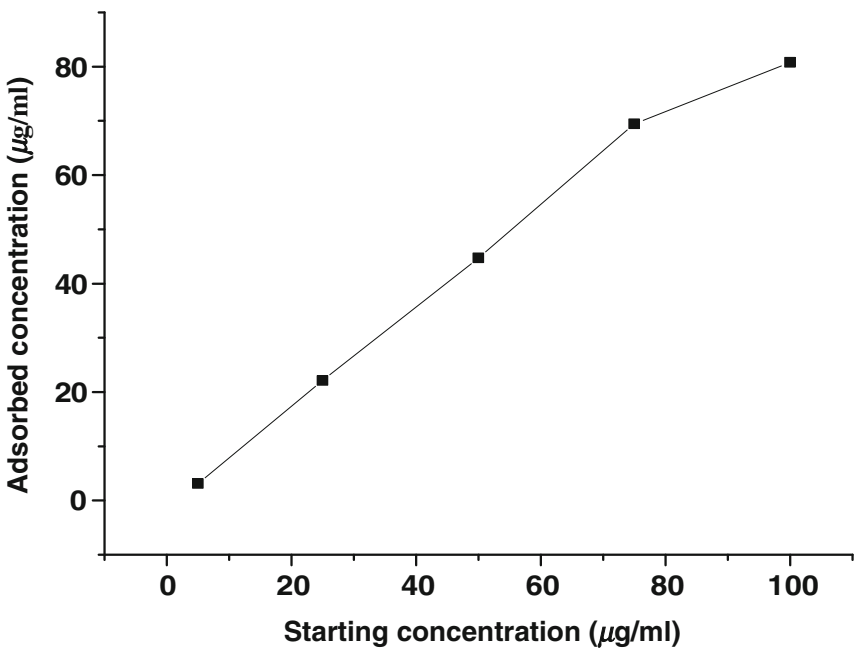

Figure 4. Loading capacity of plasmid DNA with varying concentrations.

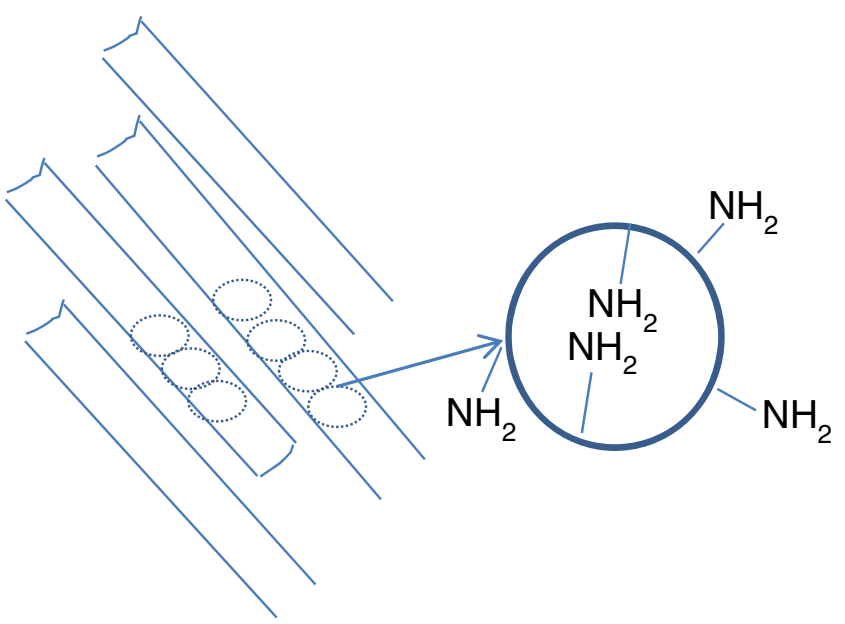

Figure 5. Schematic showing amine-functionalized pores on silica nanorods.

Silica-based nanoparticles, surface functionalized with cationic groups have been reported to electrostatically bind DNA. However due to the low external surface area, the uptake is limited. MSN's (mesoporous silica nanoparticles), on the contrary with high surface area and pore volume can immobilize larger quantities of DNA required for drug delivery. We observe that the loading capacity of the plasmid DNA on the silica mesostructure increased linearly as we increase the concentration of DNA in an aqueous solution (figure 4).

The large surface area and the controlled pore diameters have made mesoporous silica attractive as a potential delivery agent for guest molecules, which can be easily adsorbed into the silica pores by ion-exchange or covalent bonding. Since DNA contains multiple phosphate sugar groups, at physiological conditions the entire molecule behaves as a large, negatively charged polyelectrolyte. Generally, silica surfaces are negatively charged and for an effective binding 
Table 1. Zeta potential measurements.

\begin{tabular}{lc}
\hline Sample & Zeta potential $(\mathrm{mV})$ \\
\hline Bare silica & $18.2 \mathrm{mV}$ \\
DNA/silica & $-11.2 \mathrm{mV}$ \\
\hline
\end{tabular}

of DNA, charge mediation is required. Ionic salts or surface functionalization using aminopropyl groups are known to promote DNA adsorption onto silica surfaces by creating positive charge by grafting cationic linkers to the outer surface of silica nanoparticles in order to increase the uptake of polyelectrolytes (He et al 2003; Radu et al 2004).

In our study, we have used cationic surfactant to synthesize mesoporous silica. On keeping the mesoporous silica in contact with the DNA solutions, we observe a maximum loading of $8 \mu \mathrm{g} \mathrm{mg}^{-1}$ of the silica. This is double the amount as compared to $4.3 \mu \mathrm{g} \mathrm{mg}^{-1}$ of DNA that is loaded for the cation-linked mesoporous silica (Solberg and Landry 2006). The zeta potential measurements of the bare sample show a positive surface charge which could be possibly due to the presence of the cationic surfactants on the surface of the nanorod (table 1). This helps in the adsorption of the negatively-charged plasmid onto the pores of the nanorod. The loading capacity in these nanorods is much higher than the one observed by Gao et al (2009). In their study who have reported a maximum loading capacity of $5.1 \mu \mathrm{g} \mathrm{mg}^{-1}$ for the amino-functionalized silica whereas no adsorption occurred on the non-functionalized counterparts (Gao et al 2009).

We have also studied the effect of amine groups on the loading capacity of DNA. The amino-functionalized silica nanostructures were also dispersed in an aqueous solution of varying concentration of $\mathrm{p}$ Blue script $\mathrm{KS}^{+}$. We observe that the amount of the plasmid DNA adsorbed on the aminefunctionalized silica is somewhat less than that observed for the bare particle. It appears that the functionalization process results in the grafting of amine groups on the surface as well as at the entrance of the pore (figure 5). This results in the blocking of the pore entrances thereby reducing the amount of DNA adsorbed. The maximum amount of DNA loaded for the amine-functionalized silica is $\sim 7 \mu \mathrm{g} \mathrm{mg}^{-1}$.

The major challenge in the field of drug delivery lies in its ability to deliver drugs specifically to the diseased cells. The nanoparticulate drug carriers have the potential to cross physiological barriers and access different tissues, and also be provided in a targetable form (by surface-modification) aimed at enhancing cell specificity of the carrier. Larger size of these nanostructures restricts its use for transfection. However we are trying to optimize the conditions to obtain smaller-sized nanorods.

\section{Conclusions}

Silica nanorods synthesized using reverse micellar method has proved to be a useful material for the encapsulation of DNA. With high doses of plasmid loaded onto these silica mesostructures, they can be effectively functionalized for specific and targeted drug delivery. The advantage associated with these silica nanostructures is that the requisite amount of plasmid may be delivered at the affected site. Thus, we believe that the reverse micellar method using cationic surfactants offers a simple route to synthesize high-surface area nanorods that can load large quantities of plasmid DNA.

\section{References}

Chen A M, Zhang M, Wei D G, Stueber D, Taratula O, Minko T and He H X 2009 Small 52673

Choy J H, Kwak S Y, Jeong Y J and Park J S 2000 Angew. Chem. Int. Ed. 394041

Deere J, Magner E, Wall J G and Hodnett B K 2001 Chem. Commun. 5465

Fujiwara M, Yamamoto F, Okamoto K, Shiokawa K and Nomura R 2005 Anal. Chem. 778138

Gao F, Botello P, Corma A, Blesa J and Dong L 2009 J. Phys. Chem. B113 6

He X X et al 2003 J. Am. Chem. Soc. 1257168

Kisler J M, Dahler A, Stevens G W and Connor A J O 2001 Micropor. Mesopor. Mater. 44769

Kumar M N V R et al 2004 J. Nanosci. Nanotechnol. 4876

Meng H A, Liong M, Xia T A, Li Z X, Ji Z X, Zink J I and Nel A E 2010 ACS Nano. 44539

Pearce M E, Mai H Q, Lee N, Larsen S C and Salem A K 2008 Nanotechnology 19175103

Radu D R, Lai C Y, Jeftinija K, Rowe E W, Jeftinija S and Lin V S Y 2004 J. Am. Chem. Soc. 12613216

Roy I, Ohulchanskyy T Y, Bharali D J, Pudavar H E, Mistretta R A, Kaur N and Prasad P N 2005 Proc. Nat. Acad. Sci. USA 102279

Solberg S M and Landry C C 2006 J. Phys. Chem. B110 15261

Torney F, Trewyn B G, Lin V S Y and Wang K 2007 Nat. Nanotechnol. 2295

Zhu Y F, Shi J L, Shen W H, Chen H R, Dong X P and Ruan M L 2005 Nanotechnology 162633 